\title{
Noise Tolerant Descriptor for Texture Classification
}

\author{
Thanh Phuong Nguyen*, Antoine Manzanera* \\ * U2IS Robotics and Vision, ENSTA ParisTech, Université Paris-Saclay \\ 828 Boulevard des Maréchaux, 91762, Palaiseau CEDEX, France \\ e-mail: \{thanh-phuong.nguyen,antoine.manzanera\}@ensta-paristech.fr
}

\begin{abstract}
Among many texture descriptors, the LBP-based representation emerged as an attractive approach thanks to its low complexity and effectiveness. Many variants have been proposed to deal with several limitations of the basic approach like the small spatial support or the noise sensitivity. This paper presents a new method to construct an effective texture descriptor addressing those limitations by combining three features: (1) a circular average filter is applied before calculating the Complemented Local Binary Pattern (CLBP), (2) the histogram of CLBPs is calculated by weighting the contribution of every local pattern according to the gradient magnitude, and (3) the image features are calculated at different scales using a pyramidal framework. An efficient calculation of the pyramid using integral images, together with a simple construction of the multi-scale histogram based on concatenation, make the proposed approach both fast and memory efficient. Experimental results on different texture classification databases show the good results of the method, and its excellent noise robustness, compared to recent LBP-based methods.
\end{abstract}

Index Terms-Local binary pattern, texture classification, multi-resolution, noise robustness.

\section{INTRODUCTION}

Texture analysis is a very active research topic in computer vision and pattern recognition. A large number of models have been proposed [1] for texture classification. One of the most popular approaches is based on feature distribution with Local Binary Pattern (LBP), proposed by Ojala et al. [2], [3]. Thanks to its low computational cost and invariance to illumination, LBP is widely considered as an efficient descriptor for capturing local properties of images. Besides, thanks to the flexibility of the LBP framework, many variants have been proposed to fit a specific problem or to improve the robustness and discriminative power.

The two main limitations of the classic LBP codes are: (1) they capture only local features of images, and (2) they are sensitive to noise and/or uniform regions of image. Many aspects have been considered to overcome these limitations. Pre-processing steps, like Gabor filters [4] are widely used for both capturing more spatial information and decreasing the noise. Different neighborhood topologies have been used, such as elliptical neighborhood [5], three-patch or four-patch approach [6] to exploit anisotropic information. Multi-resolution or multi-structure approaches [7]-[11] were considered to gather information at larger scales. In encoding step, three values $(-1,0,1)$ were used in Local Ternary Pattern [12] to address the sensitivity to near constant image areas. Liao [13] coded only the most frequent patterns to increase the robustness. Guo et al. used complementary component about magnitude information [14] and also variance [15] to enhance the descriptive power and then improve the texture classification.

We propose a new approach to improve discrimination power of the classic LBP while enhacing its robustness to noise and near uniform regions. It combines spatial filtering and adaptive construction of the LBP histogram, which is weighted according to the gradient magnitude, with a multi-resolution analysis. Thanks to efficient calculation of the pyramid and construction of the multiscale histogram, it provides a fast and memory efficient texture descriptor. Its performance and noise robustness are evaluated on three texture data sets and compared with other state-of-the-art LBP-based descriptors. The next section recalls the most relevant related works. Section III introduces the proposed descriptor, and Section IV evaluates our method and compares it to state-of-the-art descriptors for the texture classification task.

\section{RELATED WORKS}

\section{A. $L B P$ and $C L B P$}

The LBP operator [2], [3] was introduced to represent local relations between a pixel and its neighbors by a binary code. The basic LBP is a P-bit code described as follows:

$$
L B P_{P, R}^{p}=s\left(g_{p}-g_{c}\right), \quad s(x)= \begin{cases}1, & x \geq 0 \\ 0, & x<0\end{cases}
$$

where $g_{c}$ denotes the gray value of the center pixel and $g_{p}$ $(p=0, \ldots, P-1)$ the gray value of the $P$ neighbor pixels on a circle of radius $R$. If $\left(x_{c}, y_{c}\right)$ are the coordinate of the center pixel, then the coordinates of its neighbors are $\left(x_{c}+\right.$ $\left.R \cos (2 \pi p / P), y_{c}+R \sin (2 \pi p / P)\right)$. The gray values $g_{p}$ are estimated by interpolation. The uniformity measure of a LBP is defined as follows:

$$
U\left(\operatorname{LBP}_{P, R}\right)=\sum_{p=1}^{P}\left|\operatorname{LBP}_{P, R}^{p}-\operatorname{LBP}_{P, R}^{p-1}\right|,
$$

Ojala et al. [3] then proposed the rotation invariant uniform (riu2) pattern as follows:

$$
\mathrm{LBP}_{P, R}^{r i u 2}=\left\{\begin{array}{l}
\sum_{p=0}^{P-1} \operatorname{LBP}_{P, R}^{p}, \text { if } U\left(\operatorname{LBP}_{P, R}\right) \leq 2 \\
P+1, \text { otherwise. }
\end{array}\right.
$$

In texture representation, $\mathrm{LBP}_{P, R}^{r i u 2}$ are often used to reduce the feature vector length (only $P+2$ distinct values instead of 


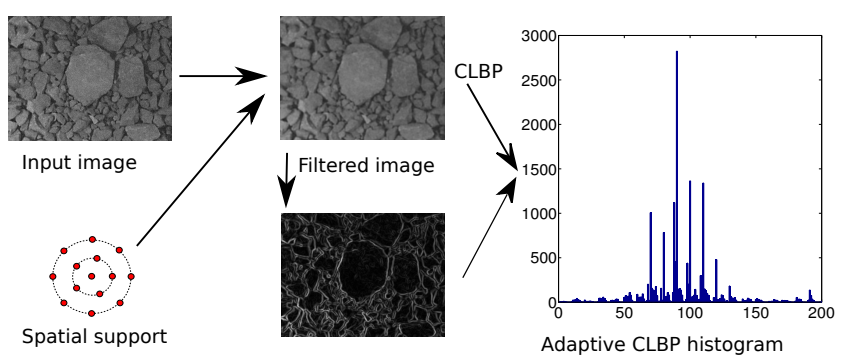

Fig. 1. Spatial filtering and calculation of the weighted histogram of CLBP codes.

$2^{P}$ ), and to make the descriptor more robust to unsignificant variations.

Guo et al. [14] significantly improved the performance of $\mathrm{LBP}_{P, R}^{r i u 2}$ by considering a completed representation (CLBP). The local differences are decomposed into two complementary components: the signs CLBP_S and the magnitudes CLBP_M In addition, the gray value of the center pixel is also converted into a binary code: CLBP_C, using a global thresholding. CLBP codes then have $2(P+2)^{2}$ distinct values.

\section{B. Noise robustness}

Different methods have been proposed to address the sensivity of LBP to noise and near uniform regions. Tan and Triggs [12] proposed local ternary patterns (LTP), using ternary $\{-1,0,1\}$ instead of binary encoding, 0 being used when the pixel difference is less than a given threshold. Ren et al. [16] proposed a mechanism to recover the corrupted patterns by encoding small difference as an uncertain bit determined later using the other bits. Mean filters have been widely used in different steps like filtering [9], thresholding [17], or sampling [18]. Song et al. [19] explored a set of local contrast patterns via global measures to handle noise effect.

\section{Multiresolution Adaptive ClBP}

This section details the proposed descriptor, whose principles are illustrated on Figures 1 and 2.

\section{A. Construction of the descriptor}

The proposed Multiresolution Adaptive Complemented LBP (MACLBP) is presented in three steps: (1) Spatial filtering, (2) Weighted histogram of CLBP codes, and (3) Multiresolution histograms.

1) Spatial filtering: Mean filters are widely used to reduce the effect of additive noise. Inspired by [9], we calculate the mean filter on a spatial support made of a union of discrete circles. The size of the support should be proportionate to the neighborhood used to calculate the LBP. Aside from noise reduction, the interest of the spatial filtering step is to capture more information in a rotation invariant manner thanks to the isotropic support.
2) Contrast weighted CLBP histogram: In the descriptors based on distribution of LBP codes, every pattern classically have the same weight, whatever its significance. However many patterns due to noise or to near uniform regions should be discarded. Considering only uniform patterns is not fully satisfying, since uniform patterns may also be due to noise and conversely, a significant part of the non uniform patterns may be highly relevant. Considering only the most frequent patterns do not avoid the contribution of spurious patterns in the most frequent bins. Our proposal to address the sensitivity to noise and near uniform regions is to weight the histogram of every pattern by a significance index corresponding to the contrast measured on the filtered image, using a Sobel filter as shown on Figure 1. This can obviously be applied with any LBP variant. In practice, CLBP [14] is chosen for its known performance in texture classification. The resulting descriptor is named Adaptive CLBP (ACLBP).

3) Pyramidal approach: To overcome the limitation of LBP to local description, a pyramidal approach is applied. The initial image is recursively reduced to half its size at each step (see Figure 2). At each resolution, ACLBP histogram is calculated. The Multiresolution Adaptive CLBP (MACLBP) descriptor is finally constructed by concatenating the ACLBP descriptors at each resolution. An important question is: should the histograms be normalized or not? Our experiments proved that the MACLBP descriptor performed better by keeping the histograms un-normalized, so that the contribution of each scale is proportional to the number of patterns, and so drops exponentially. In practice the number of resolutions is limited to 4 .

\section{B. Comments and links to other methods}

1) Use of the contrast: Gradient information has been much used to design robust local descriptors. The orientation of the gradient is the main component of descriptors like HoG [20] or POEM [21]. Gradient was also used in LBP-based variants to highlight edge structures. Yao and Chen [22] proposed an LBP-like computation on binary edges to describe texture. In Sobel-LBP [23], LBP is applied on gradient magnitude images. Weighting the LBP histogram by a contrast measure was previously done by [15] who used the gray level variance on the LBP support.

2) Multi-scale approaches: Different extensions to multiscale were proposed for LBP in the literature. Mäenpää et al. [7] combined Gaussian low-pass filters with exponentially growing circular neighborhoods. Liao et al. [9] extended the pixel-wise classic LBP to block-wise LBP by comparing average values of blocks instead of pixel gray levels. He et al. [8] used a pyramidal multi-structure based on five convolution templates including anisotropic filters. The closest approach to ours is Turtinen et al. [10], who also calculated a descriptor by concatenating histograms of LBP codes at different scales, but in their case, the image of all scales are interpolated and rescaled to the initial resolution, so that the statistical contribution of the different scales are the same. 


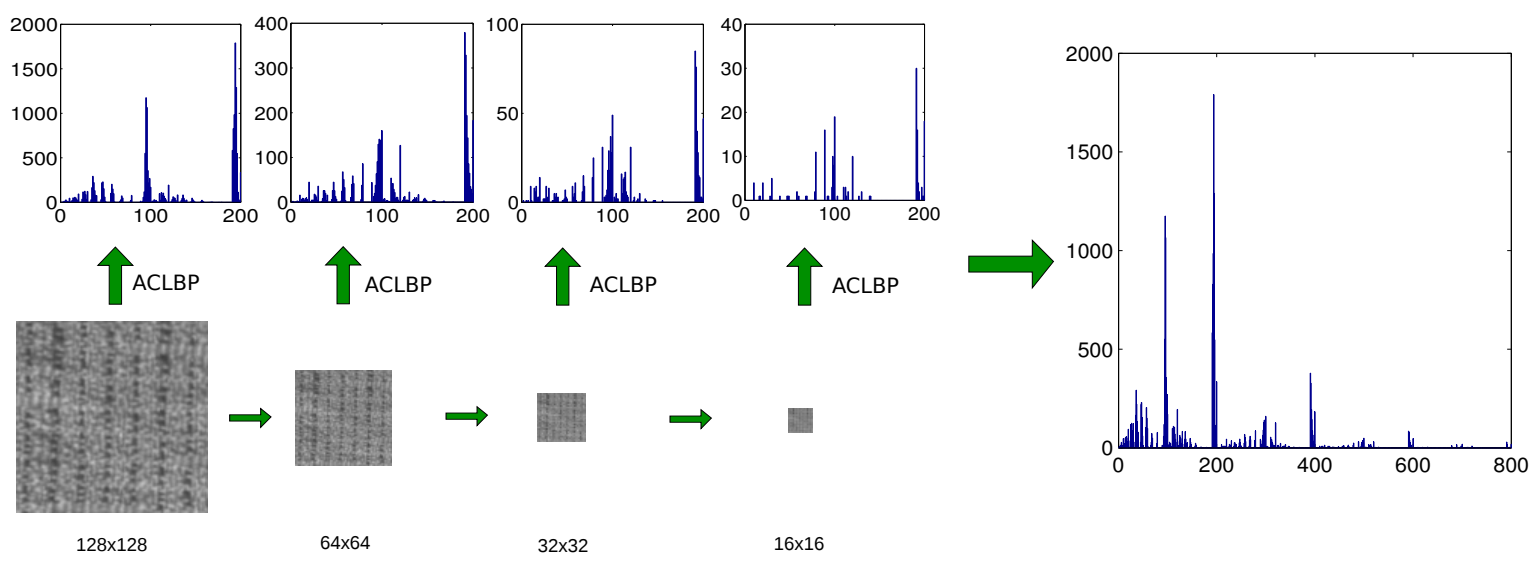

Fig. 2. From local to global features using the pyramidal approach.

3) Computational aspects: The pyramid is efficiently computed using the integral image technique similarly as [24]. Once the integral image calculated, the average value of any $2^{p} \times 2^{p}$ block is obtained by three additions and one division by a power of 2 (right bit shifting). The MACLBP descriptor is limited to a reasonable size by using the concatenation of ACLBP at the different resolution. If $P$ is the number of neighbors considered in the basic LBP, then the number of bins of the ACLBP histogram is $2(P+2)^{2}$, and so the size of the MACLBP descriptor using $S$ different scales is $2 S(P+2)^{2}$.

\section{TEXTURE CLASSIFICATION EXPERIMENTS}

\section{A. Datasets and protocols}

The effectiveness of the proposed method is assessed by a series of experiments on three large and representative databases: Outex [25], CUReT [26] and UIUC [27].

The Outex dataset contains texture images from a wide variety of real materials. We consider two common test suites, TC10 and TC12, containing 24 texture classes collected under three different illuminations and nine different rotation angles. The images at angle $0^{0}$ are chosen for training the classifier, the remaining images for testing. The CUReT (resp. UIUC) texture dataset includes 61 (resp. 25) classes with 92 (resp. 40) images in each class. The resolution of each image is $200 \times 200$ (resp. $640 \times 480$ ). These datasets contain images taken under significant viewpoint and illumination variations. In the training phase, 46 (resp. 23) images per class are randomly selected while the remainder is used for testing. The reported classification rate correspond to the average result over 100 random training/testing splits.

\section{B. Classifier}

For comparison purposes with other LBP-based descriptors, the 1-nearest neighbor classifier is used, using the $\chi^{2}$ distance between histograms to measure the similarity between two texture images.

\section{Parameter setting and component evaluation}

Different neighborhood configurations have been evaluated. In the reported results, we retained the union of two discrete
TABLE I

CONTRIBUTION OF DIFFERENT COMPONENTS ON OUTEX TC10.

\begin{tabular}{c|ccccccc} 
Noise & $\mathrm{F}$ & $\mathrm{W}$ & $\mathrm{M}$ & $\mathrm{F}+\mathrm{W}$ & $\mathrm{F}+\mathrm{M}$ & $\mathrm{W}+\mathrm{M}$ & $\mathrm{F}+\mathrm{W}+\mathrm{M}$ \\
\hline Without & 99.14 & 98.75 & 99.37 & 99.45 & 99.69 & 99.38 & 99.79 \\
\hline SNR=5 & 98.96 & 98.07 & 98.78 & 99.35 & 99.43 & 99.30 & 99.74
\end{tabular}

TABLE II

ClassificATION RATE (\%) ON OUTEX DATASET.

\begin{tabular}{c|cccc} 
Method & TC10 & TC12 $\mathrm{h}$ & TC12 $\mathrm{t}$ & Average \\
\hline \hline MACLBP & $\mathbf{9 9 . 7 9}$ & $\mathbf{9 8 . 9 8}$ & $\mathbf{9 9 . 3 5}$ & $\mathbf{9 9 . 3 7}$ \\
\hline LNIRP/LBP/DCI_PTP [30] & 99.77 & 98.87 & 98.70 & 99.11 \\
\hline LTP [12] & 98.20 & 93.59 & 89.42 & 93.74 \\
\hline DLBP+NGF [13] & 98.20 & 91.60 & 87.40 & 92.37 \\
\hline CLBP [14] & 98.93 & 95.32 & 94.53 & 96.26 \\
\hline CRLBP [17] & 99.48 & 97.57 & 97.34 & 98.14 \\
\hline LBPV [15] & 97.76 & 95.39 & 95.57 & 96.24 \\
\hline BF+CLBP [29] & 99.32 & 96.83 & 96.50 & 97.55
\end{tabular}

circles $\{(5,1),(8,2)\}$ (as seen in Figure 1) for the spatial support of the average filter and $(P, R)=(24,3)$ for the support of the LBP. Four levels of resolution were used in the pyramid. We also evaluated the contribution of the three steps composing the MACLBP descriptor as described in Section III, by testing different partial versions of the descriptor on Outex TC10 without or with additive Gaussian noise. The results can be seen in Table I, showing the complementarity of the three components (Filtering, Weighting, Multiresolution, respectively denoted $\mathrm{F}, \mathrm{W}$ and $\mathrm{M}$ in the table).

\section{Comparative evaluation}

Table II and Table III report the experimental results of our descriptor compared with different methods ${ }^{1}$, on the different datasets. It can be seen that MACLBP outperforms the stateof-the-art results on the two test suites of Outex, with an improvement up to 1-3\% with respect to recent methods, and also provides very good results on CUReT and UIUC datasets, with the best average classification rate on the two datasets.

${ }^{1}$ We reimplemented or used existing codes for the following methods: [28], [14], [3], [12], [29]. The other results are taken from the literature. 
TABLE III

CLASSIFICATION RATE (\%) ON CURET AND UIUC DATASETS.

\begin{tabular}{c|cc} 
Method & CUReT $(\mathrm{N}=46)$ & UIUC $(\mathrm{N}=20)$ \\
\hline \hline MACLBP & 97.15 & 95.58 \\
\hline CLBP [14] & 95.86 & 91.19 \\
\hline BF+CLBP [29] & 95.01 & 93.78 \\
\hline DNS+LBP $24,3[31]$ & 94.52 & \\
\hline Xu et al. [32] & \multicolumn{2}{l}{} \\
\hline BRINT2_S_M (MS9) [18] & $\mathbf{9 7 . 8 6}$ & \\
\hline Lazebnik et al. [27] & 72.5 & $\mathbf{9 6 . 0 3}$
\end{tabular}

TABLE IV

CLASSIFICATION RATE (\%) ON OUTEX TC10 AND CURET DIFFERENT SCHEMES WITH DIFFERENT NOISE LEVELS.

\begin{tabular}{|c|c|c|c|c|c|}
\hline & SNR & 30 & 15 & 10 & 5 \\
\hline \multirow{10}{*}{$\begin{array}{l}\stackrel{0}{U} \\
\qquad \\
\stackrel{\times}{\circlearrowright} \\
\stackrel{\Xi}{0}\end{array}$} & MACLBP & 99.79 & 99.76 & 99.76 & 99.74 \\
\hline & LPQ [28] & 99.14 & 99.06 & 98.88 & 98.43 \\
\hline & $\operatorname{LBP}_{P=24, R=3}$ & 94.37 & 94.22 & 93.91 & 93.57 \\
\hline & WLD [33] & 84.61 & 80.51 & 70.78 & 48.64 \\
\hline & $\mathrm{LTP}_{P=24, R=3}[12]$ & 94.76 & 94.71 & 94.37 & 93.64 \\
\hline & $\mathrm{CLBP}_{P=24, R=3}[14]$ & 99.11 & 98.91 & 98.62 & 98.23 \\
\hline & BF+CLBP [29] & 99.26 & 99.27 & 99.17 & 98.76 \\
\hline & NTLBP [34] & 96.12 & 88.85 & 80.23 & 51.09 \\
\hline & NRLBP [16] & 85.73 & 80.16 & 72.42 & 51.02 \\
\hline & $\mathrm{LCP}_{R=5}[19]$ & 96.93 & 93.54 & 90.63 & 75.05 \\
\hline \multirow{8}{*}{ 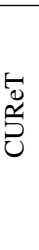 } & MACLBP & 97.12 & 96.99 & 96.87 & 96.65 \\
\hline & LBP [3] & 86.46 & 86.18 & 85.66 & 84.56 \\
\hline & CLBP [14] & 95.88 & 95.58 & 95.53 & 94.80 \\
\hline & LTP [12] & 88.55 & 87.97 & 87.86 & 86.37 \\
\hline & $\mathrm{LCP}_{R=5}[19]$ & 94.30 & 92.22 & 90.28 & 85.00 \\
\hline & ENRLBP [16] & 83.55 & 77.67 & 73.76 & 64.90 \\
\hline & DNS [31] & 83.03 & 80.00 & 77.80 & 71.60 \\
\hline & LSEP [35] & 87.58 & 83.45 & - & 72.08 \\
\hline
\end{tabular}

Table IV evaluates the noise robustness of different methods on Outex TC10 and CUReT datasets by comparing the classification rates for different noise levels (measured using SNR i.e. Signal to Noise Ratio). It can be seen that MACLBP is much more robust than the other methods: the classification rate is nearly constant when the SNR decreases, whereas it significantly drops for the other methods.

\section{CONCLUSIONS}

We have proposed an efficient variant of LBP for texture representation called MACLBP. The combined use of spatial filtering, histogram weighting based on the gradient magnitude, and multi-scale approach based on dyadic pyramid allows to increase the expressiveness of the descriptor while reducing its sensitivity to noise or near uniform regions. Thanks to efficient calculation of the pyramid and concatenation of a limited number of scale specific descriptors, MACLBP has low computational and memory footprint. The experimental results show very promising results for texture classification.

\section{REFERENCES}

[1] M. Mirmehdi, X.H. Xie, and J. Suri, Handbook of Texture Analysis, World Scientific, 2008

[2] Ojala T., Pietikäinen M., and Harwood D., "A comparative study of texture measures with classification based on featured distributions," Pattern Recognition, vol. 29(1), pp. 51-59, 1996.

[3] T. Ojala, M. Pietikäinen, and T. Mäenpää, "Multiresolution gray-scale and rotation invariant texture classification with local binary patterns," IEEE Trans. PAMI, vol. 24, pp. 971-987, 2002.
[4] F. Riaz, A. Hassan, S. Rehman, and U. Qamar, "Texture classification using rotation- and scale-invariant gabor texture features," IEEE Signal Process. Lett., vol. 20, no. 6, pp. 607-610, 2013.

[5] S. Liao and A. C. S. Chung, "Face recognition by using elongated local binary patterns with average maximum distance gradient magnitude," in $A C C V, 2007$, vol. 4844 of $L N C S$, pp. 672-679.

[6] L. Wolf, T. Hassner, and Y. Taigman, "Descriptor based methods in the wild," in Real-Life Images Workshop, (ECCV), 2008.

[7] T. Mäenpää and M. Pietikäinen, "Multi-scale binary patterns for texture analysis," in SCIA, 2003, vol. 2749 of LNCS, pp. 885-892.

[8] Y. He, N. Sang, and C. Gao, "Pyramid-based multi-structure local binary pattern for texture classification," in ACCV, 2010, vol. 6494 of LNCS, pp. 133-144.

[9] S.C. Liao, X.X. Zhu, Z. Lei, L. Zhang, and S.Z. Li, "Learning multiscale block local binary patterns for face recognition," in $I C B, 2007$, vol. 4642 of $L N C S$, pp. 828-837.

[10] M. Turtinen and M. Pietikäinen, "Contextual analysis of textured scene images," in BMVC, 2006, pp. 849-858.

[11] Y. Raja and S. Gong, "Sparse multiscale local binary patterns," in BMVC, 2006, pp. 799-808.

[12] X. Tan and B. Triggs, "Enhanced local texture feature sets for face recognition under difficult lighting conditions," IEEE Trans. Image Processing, vol. 19, no. 6, pp. 1635-1650, 2010.

[13] S. Liao, M.W.K. Law, and A.C.S. Chung, "Dominant local binary patterns for texture classification," IEEE Trans. Image Processing, vol. 18(5), pp. 1107-1118, 2009.

[14] Z. Guo, Z. Zhang, and D. Zhang, "A completed modeling of local binary pattern operator for texture classification," IEEE Trans. Image Processing, vol. 19(6), pp. 1657-1663, 2010.

[15] Z. Guo, L. Zhang, and D. Zhang, "Rotation invariant texture classification using LBP variance (LBPV) with global matching," Pattern Recognition, vol. 43(3), pp. 706-719, 2010.

[16] J. Ren, X. Jiang, and J. Yuan, "Noise-resistant local binary pattern with an embedded error-correction mechanism," IEEE Trans. Image Processing, vol. 22, no. 10, pp. 4049-4060, 2013.

[17] Y. Zhao, Jia W., R.-X. Hu, and H. Min, "Completed robust local binary pattern for texture classification," Neurocomputing, vol. 106, pp. 68-76, 2013.

[18] L. Liu, Y. Long, P.W Fieguth, S. Lao, and G. Zhao, "BRINT: Binary Rotation Invariant and Noise Tolerant Texture Classification," IEEE Trans. Image Processing, vol. 23, no. 7, pp. 3071-3084, 2014.

[19] T. Song, H. Li, F. Meng, Q. Wu, B. Luo, B. Zeng, and M. Gabbouj, "Noise-robust texture description using local contrast patterns via global measures," IEEE Signal Process. Lett., vol. 21, no. 1, pp. 93-96, 2014.

[20] N. Dalal and B. Triggs, "Histograms of oriented gradients for human detection," in CVPR, 2005, pp. 886-893.

[21] N.-S. Vu, H. M. Dee, and A. Caplier, "Face recognition using the POEM descriptor," Pattern Recognition, vol. 45, no. 7, pp. 2478-2488, 2012.

[22] C.H. Yao and S.Y. Chen, "Retrieval of translated, rotated and scaled color textures," Pattern Recognition, vol. 36, no. 4, pp. 913929, 2003.

[23] S. Zhao, Y. Gao, and B. Zhang, "Sobel-LBP," in ICPR, 2008, p. 21442147.

[24] P. Viola and M. J. Jones, "Robust real-time face detection," IJCV, vol. 57, pp. 137-154, 2004.

[25] T. Ojala, Mäenpää, T., Pietikäinen M., J. Viertola, J. Kyllönen, and S. Huovinen, "Outex - new framework for empirical evaluation of texture analysis algorithms," in ICPR, 2002, pp. 701-706.

[26] K. J. Dana, B. van Ginneken, S. K. Nayar, and J. J. Koenderink, "Reflectance and texture of real-world surfaces," ACM Trans. Graph. vol. 18, pp. 1-34, 1999.

[27] S. Lazebnik, C. Schmid, and J. Ponce, "A sparse texture representation using local affine regions," IEEE Trans. PAMI, vol. 27, no. 8, pp. 12651278, 2005.

[28] E. Rahtu, J. Heikkilä, V. Ojansivu, and T. Ahonen, "Local phase quantization for blur-insensitive image analysis," Image Vision Comput. vol. 30, no. 8, pp. 501-512, 2012.

[29] N.-S. Vu, T.P. Nguyen, and G. Christophe, "Improving texture categorization with biologically-inspired filtering," Image Vision Comput., vol. 32, no. 6-7, pp. 424-436, 2014.

[30] K. Wang, C.-E. Bichot, C. Zhu, and B. Li, "Pixel to patch sampling structure and local neighboring intensity relationship patterns for texture classification.," IEEE Signal Process. Lett., vol. 20, no. 9, pp. 853-856, 2013. 
[31] F. M. Khellah, "Texture classification using dominant neighborhood structure," IEEE Trans. Image Processing, vol. 20, no. 11, pp. 32703279, 2011.

[32] Y. Xu, H. Ji, and C. Fermüller, "Viewpoint invariant texture description using fractal analysis," IJCV, vol. 83, no. 1, pp. 85-100, 2009.

[33] J. Chen, C. Shan, C. He, G. Zhao, M. Pietikäinen, X. Chen, and W. Gao, "WLD: A Robust Local Image Descriptor," IEEE Trans. PAMI, vol. 32, no. 9, pp. 1705-1720, 2010.

[34] A. Fathi and A. R. Naghsh-Nilchi, "Noise tolerant local binary pattern operator for efficient texture analysis," Pattern Recognition Letters, vol. 33, no. 9, pp. 1093-1100, 2012.

[35] J. He, H. Ji, and X. Yang, "Rotation invariant texture descriptor using local shearlet-based energy histograms.," IEEE Signal Process. Lett., vol. 20, no. 9, pp. 905-908, 2013 . 